\title{
Analysis of Homeopathic Genus Epidemicus for COVID-19 Patients in Spain
}

\author{
Marc Lluís Clapers ${ }^{10} \quad$ Manuela Velat $^{2}$ Josep Maria Clapers ${ }^{3}$ Joan Vidal-Jové ${ }^{4}$ Joan Mora ${ }^{5}$ \\ 1 Departamento de Clínica of Academia Medico Homeopática de \\ Barcelona (AMHB), Calle Corcega $3702^{\circ} 2^{\mathrm{a}}$, Barcelona, Spain \\ ${ }^{2}$ Departamento de Clínica of Academia Medico Homeopática de \\ Barcelona (AMHB), Plaça Mons, 1, Barcelona, Spain \\ ${ }^{3}$ Clapers Homeopatia, Academia Medico Homeopática de Barcelona \\ (AMHB), Calle Corcega $3702^{\circ} 2^{a}$, Barcelona, Spain \\ ${ }^{4}$ Institut Khuab. Plaça Mons, 1, Barcelona, Spain \\ ${ }^{5}$ Departamento de Clínica of Academia Medico Homeopática de \\ Barcelona (AMHB), Calle Barcelona $422^{\circ}$, Girona, Spain \\ Homeopathy 2021;110:236-243.

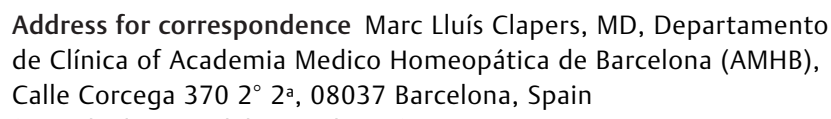

Address for correspondence Marc Lluís Clapers, MD, Departamento de Clínica of Academia Medico Homeopática de Barcelona (AMHB), Calle Corcega $3702^{\circ} 2^{\text {a }}, 08037$ Barcelona, Spain \\ (e-mail: clapersvidal@gmail.com).
}

\begin{abstract}
Keywords

- genus epidemicus

- COVID-19

- homeopathy

Background Homeopathy has had documented success treating epidemics in the last two centuries. We aimed to obtain a clear homeopathic clinical picture of coronavirus disease 2019 (COVID-19) and postulate the genus epidemicus of the disease in order to inform and enhance future treatment and prophylaxis options.

Methods We conducted a prospective case series study, collecting data from 19 homeopaths in Catalonia, Spain, from patients who presented with fever and/or cough and/or breathlessness and/or confirmed COVID-19 infection or close contact with a confirmed case. We included 107 patients, and data were recorded through a checklist questionnaire on the day of the case analysis and at day 10-15 after commencing treatment. Symptoms were collected and analyzed with the help of homeopathic repertories.

Results A total of 103 cases were mild or moderate; four were severe. The severe cases were excluded from the analysis and the 103 mild and moderate cases were analyzed and a clear overall clinical picture with mental, general, and particular symptoms was achieved. Eighty-eight cases had a complete recorded follow-up. The most prescribed medicines were Bry, Ars, Phos and Gels, whilst those with the best rates of good response were Sulph, Puls and Bry. Time to full recovery after homeopathic treatment ranged from 3.5 to 14.4 days, depending on the medicine used. The potency 200 c was associated with faster rates of full recovery and a lesser need to change remedy.

Conclusion We have defined the symptomatic homeopathic characteristics of mild and moderate COVID-19 in Spain and established a set of medicines that might be useful to consider as effective genus epidemicus.
\end{abstract}

received

October 13, 2020

accepted after revision

January 25, 2021

published online

May 25, 2021 (c) 2021. The Faculty of Homeopathy.

All rights reserved.

Georg Thieme Verlag KG,

Rüdigerstraße 14,

70469 Stuttgart, Germany
DOI https://doi.org/

10.1055/s-0041-1727204. ISSN 1475-4916. 


\section{Introduction}

Coronavirus disease 2019 (COVID-19), caused by the severe acute respiratory syndrome coronavirus 2 (SARS-CoV-2), has brought about the worst pandemic in recent decades, with tens of millions having been infected worldwide and over one million associated deaths. Though the infection is mostly asymptomatic (80\%), some individuals present bilateral viral pneumonia with or without respiratory distress, and a few individuals present a severe complex virus-mediated systemic disease, with inflammation and hypercoagulability affecting any organ and whose physiopathology is not yet well established. ${ }^{1}$ The high transmission rate of the virus and the severity of symptoms have generated a global health crisis.

There are some studies showing that homeopathy could be a good therapeutic tool in epidemic diseases. ${ }^{2-12}$ Hahnemann himself had some experience with the cholera epidemics in the early 19th century in Europe: in the sixth edition of the Organon of Medicine, he explains how to manage epidemic diseases with homeopathic treatment, making it very clear that each of them is different from the previous one, even if it is the same pathogen. Through careful observation of a certain number of cases, the homeopathic physician can analyze the "genus epidemicus", an image of the set of symptoms that all the patients present, to select one or more similar homeopathic medicines suitable for the treatment of the majority of people suffering from that epidemic disease. ${ }^{13,14}$ Hahnemann went further and even postulated that this same medicine would also be useful to prevent symptomatic disease in individuals.

The Clinical Department of the Academia Medico Homeopática de Barcelona (AMHB) launched a study in March 2020 to collect clinical cases of COVID-19 who were treated with homeopathy. The study was conducted by local homeopaths, aiming to determine the homeopathic symptomatic characteristics of the COVID-19 outbreak and to identify a group of epidemic medicines or a genus epidemicus that would be useful to treat most patients. A secondary objective was to analyze the response to the homeopathic treatment.

\section{Methods}

The present study is a case series of confirmed and suspected COVID-19 cases collected in Spain. It is an open, prospective, multi-centered, non-comparative cohort of cases. The study obtained Institutional Review Board approval by the AMHB on March 30th, 2020. All subjects gave informed consent to data collection and data were anonymized at source.

Cases were collected from the private practices of homeopathic physicians who are the members of the AMHB. On March $13^{\text {th }}, 2020$ (day 0 of our study), all AMHB members (approximately 190) received an email explaining the study, its objectives, and two checklist documents (-Supplementary Files 1 and 2, available online only) to enable data collection from patients, as well as informing them about the inclusion and exclusion criteria. An email address was also provided to every associate to centralize the case data collection (email and AMHB database).
Any patient consulting a homeopath member with symptoms of fever, cough, or respiratory distress, and with high clinical suspicion of COVID-19 or close contact with a confirmed diagnosis of COVID-19, or with diagnostic confirmation by PCR, could be included in the study after providing informed consent.

Two questionnaire checklists have been used: Q1 and Q2 (-Supplementary Files $\mathbf{1}$ and 2, available online only). Q1 is the initial questionnaire, which collects personal data and symptoms, preferably completed by the patient, and with a free-text section to characterize symptoms, sensations, and others. A later section allows the patient to select the modalities of each of the three predominant symptoms in the clinical picture (fever, cough, and respiratory difficulty). Finally, there is a section to specify any risk factors for severe disease and there is also a space to register homeopathic prescription (medicine, potency, doses). Q2 should be completed at 10-15 days after starting the treatment, whenever the outcome was favorable, or earlier whenever the patient needed a change of prescription if there had been no improvement (second homeopathic prescription).

Data obtained from the questionnaires have been transferred to a database from which the statistical analysis has been done (presented in Results).

To evaluate each prescription made, we have used the descriptive term "good response" if it produced amelioration and no other remedies were prescribed for a full recovery. If a change of remedy was needed, although the first prescription ameliorated some of the symptoms, the prescription was evaluated as "not good response".

\section{Results}

On June 6th, 2020, the epidemic curve flattened in our region. The new cases being fewer and having collected more than 100 cases, we decided to finish data collection and start analysis of the data to share them with the scientific community. We present here the findings for medicines used, together with symptoms, sensations, and follow-up of confirmed or highly suspected COVID-19 cases ( - Table $\mathbf{1}$ ).

Data from 107 clinical cases were provided by 19 homeopathic local doctors. All cases came from Spanish individuals, mostly from Catalonia region (98\%). Using the WHO COVID-19 disease severity classification, ${ }^{1} 90$ (84.1\%) of our cases could be classified as mild (fever, flu-like symptoms, and negative radiology test $[\mathrm{Rx}]), 13(12.1 \%)$ as moderate (same plus $\mathrm{Rx}$ positive and mild hypoxia and dyspnea), two (1.9\%) as severe (hypoxia and dyspnea, oxygen needed, and hospitalization), and two (1.9\%) as critical (severe acute respiratory syndrome). It should be said that 34 of the cases classified as mild (30\% of total) were patients between mild and moderate categories; those patients had dyspnea or breathlessness with no $\mathrm{Rx}$ findings or with no Rx performed. Seven patients (6.5\%) required hospitalization at some point and two patients died (1.9\%).

In order to simplify our data analysis and to enable stronger conclusions on the genus epidemicus or set of homeopathic medicines most likely adequate to treat these 
Table 1 Homeopathic medicines used

\begin{tabular}{|l|l|l|l|l|l|l|}
\hline $\begin{array}{l}\text { Homeopathic } \\
\text { remedy }\end{array}$ & $\begin{array}{l}\text { Number of } \\
\text { prescriptions }\end{array}$ & $\begin{array}{l}\text { Good } \\
\text { response } \\
\text { cases }\end{array}$ & $\begin{array}{l}\text { Dyspnea, chest } \\
\text { pain, or } \\
\text { moderate cases }\end{array}$ & $\begin{array}{l}\text { Good response in } \\
\text { dyspnea, chest pain, } \\
\text { or moderate cases (\%) }\end{array}$ & $\begin{array}{l}\text { Time to full } \\
\text { recovery since } \\
\text { prescription (days) }\end{array}$ & $\begin{array}{l}\text { Used in first } \\
\text { prescription }\end{array}$ \\
\hline Bry & 29 & $21(72 \%)$ & 14 & $60 \%$ & 8.2 & 22 \\
\hline Ars & 20 & $10(50 \%)$ & 7 & $43 \%$ & 13.7 & 20 \\
\hline Phos & 16 & $9(56 \%)$ & 9 & $33 \%$ & 14.4 & 10 \\
\hline Gels & 10 & $2(20 \%)$ & 1 & $100 \%$ & 10.5 & 6 \\
\hline Camph & 7 & $3(43 \%)$ & 6 & $50 \%$ & 11 & 2 \\
\hline Sulph & 6 & $6(100 \%)$ & 4 & $100 \%$ & 3.5 & 3 \\
\hline Bell & 6 & $4(67 \%)$ & 1 & $0 \%$ & 5.75 & 4 \\
\hline Puls & 5 & $4(85 \%)$ & 0 & - & 6.25 & 4 \\
\hline Chin & 4 & $0(0 \%)$ & 2 & $0 \%$ & - & 1 \\
\hline Nux-m & 4 & $3(75 \%)$ & 2 & $50 \%$ & 4.3 & 0 \\
\hline Nux-v & 4 & $2(50 \%)$ & 4 & $50 \%$ & 1.5 & 1 \\
\hline Ant-t & 4 & $2(50 \%)$ & 1 & $100 \%$ & 15 & 3 \\
\hline
\end{tabular}

Other remedies prescribed: Sil, Lach, Grin, Nat-m, Lat-m, Thuj, Kali-c, Spong, Rhus-t, Carb-v, Sarcol-ac, Tritic-vg, Verat, Lyc, Kali-s, Chinin-m, Petr, Bamb-a, Ferr-p, Abrot, Ph-ac, Caps, Mag-c, Cypra-eg, Moly, Arn, Caust, Sam-ox, Merc, Am-c, Calc.

patients, we have analyzed the data only from the 103 patients with mild or moderate disease. We have excluded the four patients with severe or critical disease because their small numbers could invalidate the extrapolation of findings.

Most of the patients were women (76; 73\%). The average age was 49.2 years (range, 3-72 years). Twenty-five cases (24\%) had been confirmed by PCR as COVID-19 and another 50 (49\%) had a close contact with a PCR-confirmed patient; at the end, $73 \%$ of the cases collected were confirmed COVID-19 cases or highly susceptible due to a close contact with a positive patient. Three cases were tested negative, despite highly compatible symptoms (possible false negative).

Radiology tests ( $\mathrm{Rx})$ were conducted in 18 cases: 17 simple chest X-rays and one computed tomography. Twelve cases (12\%) had pathological findings: four bilateral findings (one in lower lobes); five in the lower lobe right lung; one in the left lung; two non-specified.

The checklist included the main co-morbidities described as negative factors in COVID-19 prognostics ${ }^{1}: 11$ (10.7\%) patients had chronic pulmonary disease, eight (7.7\%) obesity, and three $(2.9 \%)$ cardiovascular disease; no one had diabetes.

\section{Fever, Cough, and Respiratory Distress}

The cases collected showed a fever mostly at night, with two major temperature peak hours, 7 p.m. and 11 p.m. Most patients reported feeling cold and chilly $(60 ; 58 \%)$, with desire to be covered $(49 ; 47.5 \%)$ and worsening when uncovered. Thirty (29\%) of the patients referred to cold body parts, such as feet and hands, with icy coldness in some cases. Chills were frequent (47\%) and it was common to see fever without perspiration or with night perspiration. Likewise, most referred to thirstlessness and, in fewer cases, loss of appetite.

In half of the cases there was little cough or no cough (45; $44 \%$ ). When it was present, it was dry cough, predominantly at night. As for its modalities, it worsened with inspiration and deep inspiration, by exposure to cold air and especially when speaking and with movement; it improved by changing position, sleeping, and by lying down. Those modalities seem to be more general than specific to the cough itself, and in most of the cases cough was not unbearable or generating discomfort to the patient (see also Discussion).

Forty-three patients $(41.7 \%$ of the cases) presented with respiratory difficulty or the sensation of suffocation. Similar to fever and cough, it was predominant at night, worsened especially by movement and talking, but also by standing, eating, coughing, or by breathing in deeply. It improved when lying down or when changing position.

\section{Concomitants}

Regarding the concomitants, we should highlight weakness and fatigue (89\%), muscle pain (62\%) and headache (55\%) above the rest. Diarrhea (28\%) was also a common symptom. A few patients (3.9\%) presented extreme fatigue, with difficulty in holding up their own head or other parts of the body.

\section{General Symptoms}

The most prominent symptoms and general modalities were great desire to lie down in bed (13\%) and sleepiness (10\%). Improvement from sleep, as well as extreme weakness, was also present. There was desire for fruit, juices, or fresh things (8.7\%) and improvement by warm drinks (13\%). General worsening from talking was notable, aggravating cough, and respiratory difficulty. Some patients reported feeling worse by noise or by tight clothes.

\section{Mental Symptoms}

The most relevant symptom in this section is "anxiety about one's own health" (17.5\%), with frequent fear of sickness, death, or hospitalization. Anxiety in general was worse at night with, in the most symptomatic cases, a very specific 
and repeated symptom of fear of suffocation or dying from suffocation (11 cases, $10.7 \%$ ). Other fears and concerns have also been collected: anxiety about family members, fear of being alone, fear of poverty, and fear of insanity or becoming insane.

Other mental symptoms that emerged to a lesser extent were irritability (6\%), lack of concentration, dullness and slow thinking ( $9 \%$ globally), or apathy.

\section{Local Symptoms and Sensations}

Due to their frequency and relevance, we must underline loss or alteration of taste $(21 \%)$ and loss of smell $(20 \%)$, or the presence of both (13.6\%), as being characteristic of COVID-19.

With regard to pulmonary symptoms and sensations, the most relevant were chest oppression or tightness (14.5\%), mainly focused on the sternum; chest pain (7.7\%), usually stitching or lancinating; and suffocation (7.7\%). Particular sensations described by patients were internal dryness, itching, heat, tickling in the lungs, air itching when entering the lungs and, with lesser frequency, burning, tiredness, fullness, soreness, and "sticky".

All along the airway path, from the nose, mouth, larynx, throat, to the trachea, the same sensations were described: dryness, itching, and burning. A cold air sensation and choking mucus in the larynx were also referred to.

Symptoms in other areas of the body were also described. The most repeated symptoms were loathing of food (5\%), internal dryness (6\%), and itching eruptions (5\%). Also, soreness in the eyes, noises in the ears, fullness of stomach, skin sensitive to touch, trembling weakness, vertigo, and palpitations were seen to a lesser degree.

\section{Symptoms Analysis}

The repertorizations presented in - Fig. 1 (Radar Opus) and -Fig. 2 (MacRepertory) provide a synthesis of the symptoms reported by patients who were analyzed and registered in the database, showing: (a) the most peculiar, like the loss of taste and/or smell; (b) the most characteristic (fever, chill, cough, difficult respiration, chest oppression) with its concomitants or its peculiarities; (c) general symptoms; (d) multi-locational sensations, ameliorations, or aggravations, such as itching or tickling sensations all through the airway, dryness over all mucous membranes, a general amelioration with lying down or a general aggravation by talking; and (e) mental symptoms (fear of suffocation and anxiety about health).

\section{Homeopathic Medicines Prescribed}

Eighty-eight cases had a complete follow-up; 15 of the 103 cases were lost to follow-up or the information provided was insufficient to evaluate the prescription. We have evaluated all prescriptions from 88 cases with complete follow-up. As stated in Methods, the "Good response" category has been given if the prescription produced amelioration and no other remedies were prescribed for a full recovery. To study better the most prescribed remedies, their effectiveness, in terms of time to full recovery and by first prescription, was evaluated separately for cases of dyspnea and/or chest pain and for all moderate cases.

Forty-three different homeopathic medicines were prescribed. As shown in -Table 1, the most prescribed were Bryonia alba, Arsenicum album, Phosphorus, Gelsemium and Camphora. The remedies that showed better results (with six or more cases collected) were Sulphur (100\% "good response"), Pulsatilla (85\%), Bryonia alba (72\%), Belladona (67\%), Phosphorus (56\%) and Arsenicum album (50\%). Gelsemium and China officinalis, though being among the most prescribed medicines, each showed a very low response in this same sub-group of cases ratio ( $20 \%$ and $0 \%$, respectively).

When lower respiratory tract symptoms were prominent (dyspnea, chest pain, and for moderate COVID-19 cases), the most prescribed medicines were Bryonia alba, Phosphorus, Arsenicum album and Camphora. The best responses in this

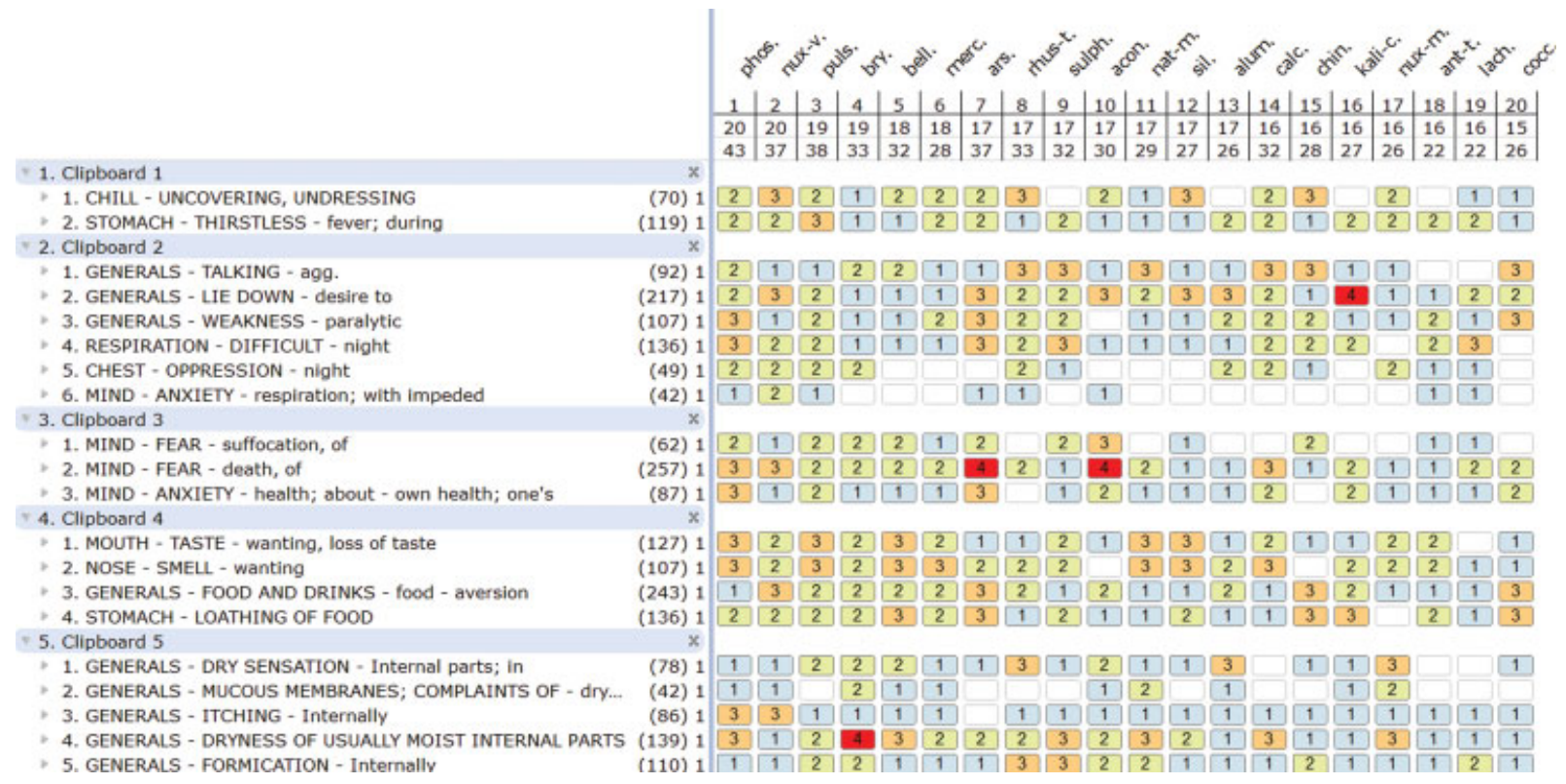

Fig. 1 Synthesis repertory-RadarOpus. ${ }^{15}$ 


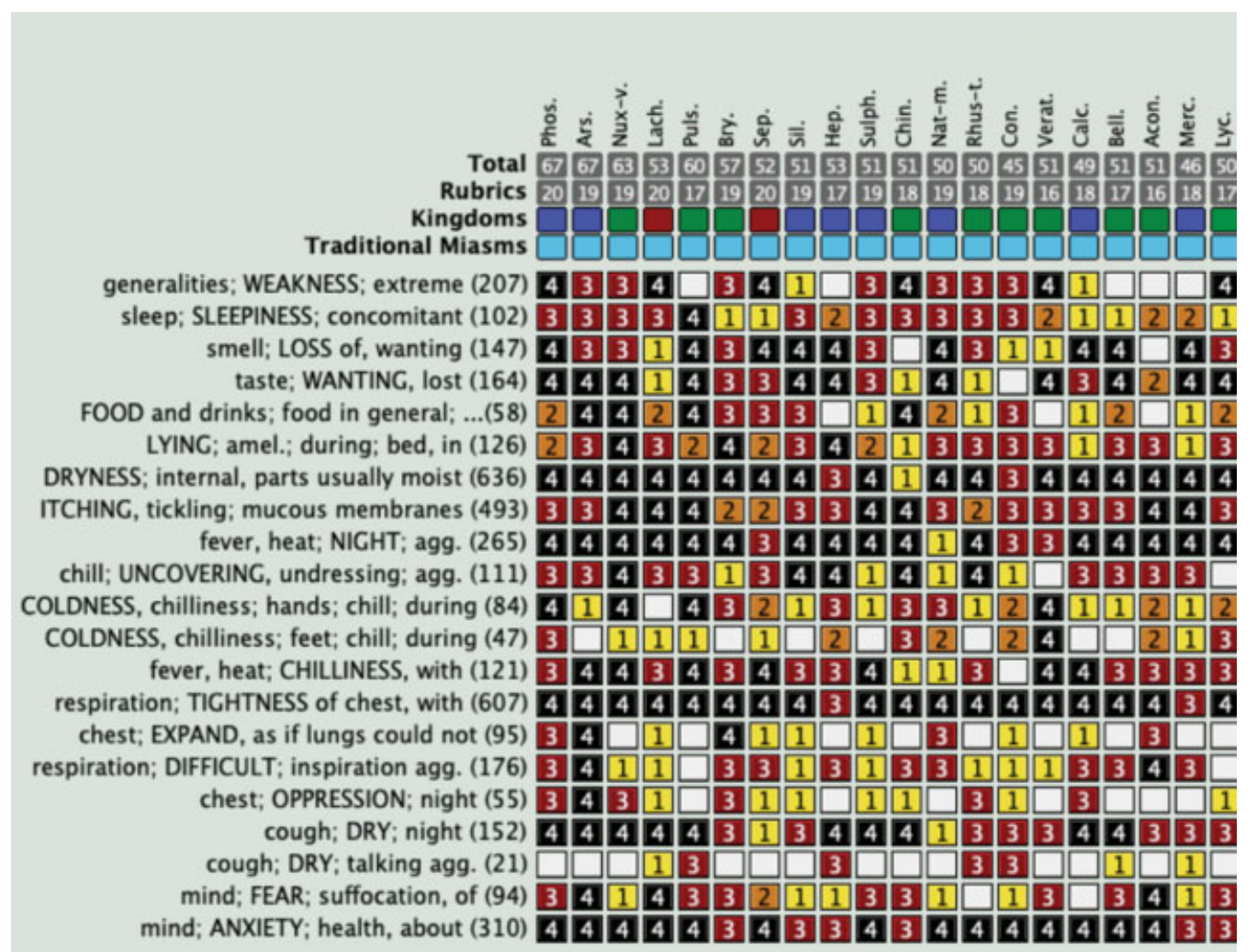

Fig. 2 Complete repertory-MacRepertory. ${ }^{16}$

same sub-group of cases were obtained (for six or more cases) with Bryonia alba (60\%), Camphora (50\%), Arsenicum (43\%) and Phosphorus (33\%).

The average time to full recovery of all 88 cases was 13.3 days (range, 1-60 days), with an average of 1.6 homeopathic medicines used per case. Full recovery time after homeopathic prescription ranged from 3.5 to 14.4 days, depending on the remedy used, and the fastest recoveries were obtained with Sulphur, Belladonna, Pulsatilla and Bryonia alba (for remedies used in more than six cases).

Some of the patients were treated using homeopathy along with conventional medicines or natural supplementation as follows: azithromycin (five cases), hydroxychloroquine (4), non-specified antibiotics (3), anti-viral drugs (3), corticosteroids (2), and vitamin $C(4)$.

With regard to potency of remedies, 30c was the most frequently used, followed by 200c and then LM potencies (-Table 2). A change of remedy or potency was needed in more than $50 \%$ of the cases that received a $30 \mathrm{c}$ potency. On the other hand, 200c potency remedies had the best ratio of good response (62\%) among the more frequently used potencies.

\section{Discussion}

To our knowledge, this is one of the largest reported collections of COVID-19 cases treated with homeopathy. ${ }^{16,17}$ Besides having some methodological, study sample or data collection bias, the results of the study seem strong enough to be extrapolated to other populations infected with a symptomatically mild or moderate COVID-19 disease, enabling us to
Table 2 Homeopathic potencies used

\begin{tabular}{|l|l|l|l|l|}
\hline Potency & Used & $\begin{array}{l}\text { Good } \\
\text { response }\end{array}$ & $\begin{array}{l}\text { Change of } \\
\text { potency is } \\
\text { needed }\end{array}$ & $\begin{array}{l}\text { Change of } \\
\text { remedy is } \\
\text { needed }\end{array}$ \\
\hline $5 c$ & 1 & - & - & 1 \\
\hline $7 c$ & 1 & 1 & - & - \\
\hline $15 c$ & 1 & - & 1 & - \\
\hline $30 c$ & 89 & 43 & 11 & 35 \\
\hline $200 c$ & 45 & 28 & 4 & 13 \\
\hline $1 \mathrm{MK}$ & 6 & 4 & - & 2 \\
\hline $10 \mathrm{MK}$ & 1 & & 1 & \\
\hline $\mathrm{LM}$ & 19 & 12 & & 7 \\
\hline
\end{tabular}

postulate a valid genus epidemicus with a candidate set of useful homeopathic medicines that could be helpful in treating or preventing further COVID-19 outbreaks.

On the various factors that could somehow limit the validity of the study, we would like to comment first about the study sample. Even though the study sample is quite heterogeneous, representing all age ranges and both sexes, it is likely to be representative only for mild and moderate cases of COVID-19. We attended only four cases of severe or critical COVID-19. Those latter cases presented with severe respiratory distress and other characteristic symptoms of advanced stages of the disease, with complex systemic involvement established. As the analysis of those four cases might have altered the validity of the results for mild or 
moderate cases, we decided to exclude them from this analysis. As commented by Waisse et $\mathrm{al}^{17}{ }^{17}$ the coronaviruses have a large symptomatic range and affect some age groups differently from others, so other studies focusing on severe disease are needed to determine its symptomatic homeopathic characteristics and its genus epidemicus.

Another bias that we may have incurred is in patient selection. In order to be included in the study, cases needed to meet specific symptomatic criteria (presence of fever, cough, and/or dyspnea) instead of allowing any symptom related to disease in confirmed COVID-19 cases. It would probably have been more rigorous if the selection criteria had been only PCR-confirmed COVID-19 cases with any presenting symptomatology, but due to scarcity of diagnostic tests at that time in our region, especially for those patients with mild disease, it was difficult to do so. As the clinically established picture elsewhere was the presence of fever, cough, or respiratory distress, we decided to focus on patients attending our practices with those symptoms in order to focus on COVID-19 and trying to exclude other diseases. This selection might have given importance to these symptoms, thereby underestimating others. Having quite a large sample size, however, we feel we have reduced the possible impact of this selection bias.

We recognize it would have been desirable to have all included cases confirmed by PCR test. At the time of data collection, there was a high community transmission rate in our region and low availability of diagnostic PCR tests, so Health Authorities' policies were advising testing only in moderate-to-severe cases of COVID-19, and mostly attending in hospital facilities. Nevertheless, 29 cases (27\%) were confirmed and another 50 (46.7\%) were in close contact with a patient with a positive PCR test. So, up to $70 \%$ of the cases collected were confirmed or were positive symptomatic contacts, which we believe is high enough to give validity to our study.

As stated in Methods, data were collected through questionnaires that were completed either by the patient or physician, but although the recommendation was their completion by the patient, most of them were completed by physicians, probably losing quality and detail in terms of wording and expressions. Furthermore, whilst the questionnaire allowed a free-writing section, it was mostly a checklist, which again might not be the best option for quality and individualization as it induces a form of response, but it is still a good means of easy collection and systematization of information about specific symptoms and their modalities.

Regardless of all those confounders and limitations, we believe that the analysis of more than 100 cases shows a welldefined clinical homeopathic picture of the mild-moderate COVID-19 disease in our region, valid for finding a set of genus epidemicus medicines, or remedies most likely to be useful. As shown in Results, the clinical picture is as follows: presents predominantly fever at night, with a sensation of chilliness, chills, cold or icy hands and/or feet, needs to be covered, perspiration is absent or nightly perspiration appears, with thirstlessness. Dry cough is common. In the respiratory domain, we have seen suffocative sensations, shortness of breath, with tightness of chest, and predominantly nocturnal, with dry, itchy, tickling, and burning sensations in the lungs and the entire respiratory mucous membranes. Mental symptoms such as anxiety at night, fear of suffocation at night or during sleep, and concern or anxiety about their own health were frequent. In the general symptoms section, we found fatigue predominantly, sometimes extreme, with great need of lying down, resting, and sleepiness. Muscle pain and headache (with no clear modalities) were usually described by patients. Diarrhea was also commonly noted. Talking worsens the patient situation generally, and more particularly cough, the suffocative sensation and the dryness. There can be loss of taste and smell and an aversion, disgust or nausea for food, improvement by hot drinks, and desire for fruit or refreshing food.

With this final breakdown of the symptoms that seem most relevant to us, we would like to emphasize some aspects. Though COVID-19 is a viral disease with clear involvement of the respiratory tract and one of the characteristic symptoms of the disease is cough, we found that cough was not so predominant among our patients, and those who present it were not able to describe any particular characteristics or modalities other than a dry cough that improves when lying down and worsens with speaking. These latter two modalities, rather than being particular to the cough itself, seem to be general modalities, and they are also referred to in association with respiratory distress, and general and local symptoms.

On the other hand, dyspnea is expressed as a feeling of suffocation, that air is not inhaled, that the lungs cannot be filled up, etc., but in most cases without tachypnea. In many patients, it seems that the patient is not able to discriminate between anxiety and respiratory distress; subjective and objective symptoms are mixed, and the patient does not know or can differentiate them. There is likely to be a component of both, but this perception reported in some cases seems relevant to us. Since we must sub-divide the symptoms by categories, we have included the feeling of suffocation as a respiratory difficulty and also as anxiety or fear of suffocation, depending on the emphasis in the patient story. Regarding mental symptoms, fear and anxiety predominate, both closely related to the feeling of suffocation, and predominantly at night or sleeping. Fear and anxiety are about death and suffocation. Also, though not so relevant, fear of becoming ill and fear of contagion are worries that perhaps we can attribute in part to the contemporary social situation of "lockdown", bad news, uncertain information, etc.

Other relevant symptoms that we would like to emphasize are the loss (or sometimes alteration) of taste and/or smell, very characteristic and particular to the current epidemic.

Likewise, about the general symptoms, we underline the tiredness, prostration and weakness expressed by the majority of patients, in some cases extreme fatigue that is a very intense, almost paralytic, weakness manifested as "I cannot even hold my head up" or "I cannot hold the phone in my hand". Conversely, the headache, whilst very frequent, does not present any particular characteristics, and so for now it has not been included in the repertorizations. 
Another symptomatic aspect that seems important to us in the analysis for the genus epidemicus is the sensations expressed by patients. In the cases collected, it seems that they are "multi-locational": that is, the same kind of sensations is experienced in different places in the body. There is predominantly dryness, which is very intense, at various levels: the mouth, throat, larynx, lungs, but also itching and burning, expressed as "the air itches on entering" (reported by two patients), "tickling of the lungs on inspiration", or "feeling that the lungs are sticking inside".

These symptoms-the most frequent and intense but also the most characteristic and particular-are the ones that should guide our analysis in the search for a genus epidemicus, a clear symptomatic picture of the COVID-19 disease in the current epidemic in our region. And the in-depth study of this genus must lead us to Materia Medica of the most similar homeopathic medicines.

As seen in the repertorizations in - Figs. $\mathbf{1}$ and 2, the remedies that appear in the first positions have also been used in the prescriptions. We must underline the remedies that appear at the top of each repertorization: Phosphorus, Nux vomica, Pulsatilla, Bryonia alba, Rhus toxicodendron, Arsenicum album, Lachesis, Sulphur. Going through the Materia Medica, we could exclude some of them, such as Rhus toxicodendron.

Bryonia alba was the most used remedy and offered an acceptably good response ratio of $72 \%$. Also, it was the most prescribed remedy for the moderate stage and for dyspnea and chest pain cases, with $60 \%$ of good response in those. The average time to full recovery since prescription was below that for all remedies (8.2 days). Though it is not the firstlisted remedy from the repertorizations, it is always among the top ten and its Materia Medica is far better correlated with the symptoms gathered from COVID-19 patients.

Phosphorus is also a remedy to be considered when treating COVID-19 patients. It appears as first-listed on both repertorizations, Complete and Synthesis, and again its Materia Medica has a good correlate with the symptom characteristics of COVID-19. However, its results after prescription have been less promising than with Bryonia, showing a good response ratio of $56 \%$ only and being the second-most prescribed remedy in moderate cases, with 33\% good response. Average time to full recovery since prescription was 14.4 days.

Sulphur has to be also considered in this group of useful remedies for COVID-19. It appears well positioned in the repertorization ranking and has shown best results in the cases collected, with $100 \%$ of good response when prescribed in our mild and moderate cases. Its average time to full recovery since prescription is also the shortest, at 3.5 days. Nevertheless, half of the Sulphur cases were the ones where this remedy was prescribed to treat persistent or convalescence symptoms.

Pulsatilla shows great potential as well. It appears in the top 5 of both repertorizations and it has a high good response ratio, around $85 \%$. It was associated with the second shortest average time for full recovery, achieved in an average of 6.25 days. However, all Pulsatilla cases were mild, and so its effectiveness in moderate cases still needs to be evaluated. The same happened with Belladonna, a remedy that also appears well positioned in repertorization and showed good results, but only in mild cases. Camphora, a remedy postulated to be useful by Sankaran's group, and even a candidate for genus epidemicus, ${ }^{18}$ does not appear in the top rankings in our study repertorizations but showed $50 \%$ good response rate in moderate cases.

Arsenicum album, a remedy recommended at the beginning of the epidemic by the AYUSH in India as genus epidemicus, ${ }^{19}$ and widely prescribed among our study patients, yielded uneven results: it showed a good response ratio of 50\% and $43 \%$ in mild and moderate cases, respectively, but it also displayed a long average time to full recovery (13.7 days). It appears well positioned in our repertorizations and it consistently has a good symptomatic correspondence with the clinical picture outlined in the study, but in some cases where it was prescribed it seemed to be highly ineffective and many patients needed a change of remedy. We recommend prescribing it with caution.

Based on the results of the symptoms analysis and the prescription outcomes, Nux vomica and Nux moschata may also be good candidates for genus epidemicus, but their case sample size is too small to make further conclusions. Other remedies like Gelsemium, widely used and recommended in other studies, ${ }^{19-21}$ and China officinalis, showed a very low response ratio, $20 \%$ and $0 \%$ respectively in our study's sample, and so their prescriptions should be considered carefully.

Given the previous repertorizations and the results obtained from the remedies used in our collected cases, which we have summarized above, it is difficult to propose just one homeopathic medicine as the genus epidemicus. However, we could probably agree on a group of remedies, as Kent said, ${ }^{14}$ with which we might treat nearly all mild and moderate cases of COVID-19 with success. Those medicines would be Bryonia alba, Phosphorus, Sulphur, Camphora and Pulsatilla.

Regarding the potencies used, we saw from our results that $30 \mathrm{c}$ was the most prescribed, but up to $60 \%$ of the times it needed a successive remedy, a higher potency, or a different medicine. 200c was frequently used too, and with better response. With these data, and though $30 \mathrm{c}$ is the most easily found potency remedy in pharmacies, it seems that using it could delay the healing process or the search for the best remedy in moderate disease cases. We suggest using, whenever it is possible, 200c remedies from first prescription to reduce as much as possible the need for changing potencies and to obtain a quicker healing response.

\section{Conclusion}

After presenting all the data and conclusions from the collected cases, we firmly believe that we have accomplished our goal, to present a clear symptomatic picture of the mild and moderate COVID-19 disease in our region. We presented also a group of remedies most suitable to treat those cases. Nevertheless, we should reflect that for each case studied and treated, the good homeopathic physician should still always individualize and prescribe the proper homeopathic medicine guided by the Law of Similars, even in treating epidemic disease, as Kent said in his Lectures. ${ }^{14}$ 
The importance of publishing this work is that its comprehensive homeopathic clinical information can reach as many homeopaths worldwide as possible, and it can be used to inform about the treatment of COVID-19 patients, taking into account that, at the time of the study, there have been no preventive or effective conventional treatments for the disease. We think homeopathy has much to contribute in reducing deaths and morbidity of this disease. Further studies are needed to investigate the role of homeopathy in severe cases of COVID-19 and in critical stages where the involvement of inflammation and hypercoagulability drive the organism to multi-organ failure.

Highlights

- We present an open, prospective, multi-centred, noncomparative cohort of cases of suspected and confirmed COVID-19 from Spain. More than 100 cases were collected.

- Symptomatic homeopathic characteristics of mild and moderate COVID-19 are defined.

- A set of remedies that could be useful as genus epidemicus are highlighted.

- Bryonia alba, Phosphorus, Sulphur, Camphora and Pulsatilla are the best genus epidemicus candidates in our region.

- The 200c potency achieved fastest rates of full recovery and the least need to change remedy.

\section{Supplementary Files}

Supplementary File 1 Questionnaire Q1. Patient symptoms.

Supplementary File 2 Questionnaire Q2. Follow-up checklist.

Conflict of Interest

None declared.

\section{Acknowledgements}

The authors wish to acknowledge the collaboration of colleagues from Academia Medica Homeopatica de Barcelona (AMHB) who shared their anonymized clinical cases with us, making this study possible.

\section{References}

1 Diaz JV, Baller A, Banerjee A, et al. Clinical management of COVID-19. WHO 2020. WHO reference number: WHO/2019-nCoV/clinical/
2020.5. Accessed September, 2020 at: https://www.who.int/publications/i/item/clinical-management-of-covid-19

2 Gonzalez-Carbajal García I. Homeopatía 100 años de historia. Madrid: Federación Española de Médicos Homeópatas (FEMH); 2004:168

3 Nicolai T. Homeopatía para las enfermedades epidémicas en los países en desarrollo. Rev Med Homeopat 2009;2:119-126

4 Jacobs J. Homeopathic prevention and management of epidemic disease. Homeopathy 2018;107:157-160

5 Frei H. H1N1 influenza epidemic 2011: experiences with polarity analysis. Spectrum of Homeopathy 2011;3:26-37

6 Rotter G. Homoeopathy and Epidemics. Possible Approacheswhat history can teach us. Graz: Liga Medicorum Homeopathica Internationalis (LMHI) Meeting; 2003

7 Van Erp M, Brands M. Homeopathic treatment of malaria in Ghana. Br Homeopath J 1996;85:66-70

8 Danno K, Rerolle F, de Sigalony S, Colas A, Terzan L, Bordet MF. China rubra for side-effects of quinine: a prospective, randomised study in pregnant women with malaria in Cotonou, Benin. Homeopathy 2014;103:165-171

9 Bracho G, Varela E, Fernández R, et al. Large-scale application of highly-diluted bacteria for leptospirosis epidemic control. Homeopathy 2010;99:156-166

10 Golden I, Bracho G. A reevaluation of the effectiveness of homoeoprophylaxis against leptospirosis in Cuba in 2007 and 2008. J Evid Based Complementary Altern Med 2014;19:155-160

11 Manchanda RK. Dengue epidemic: what can we offer? Indian J Res Homoeopathy 2015;9:137-140

12 Nair KRJ, Gopinadhan S, Kurup TNS, et al. Homoeopathic Genus Epidemicus 'Bryonia alba' as a prophylactic during an outbreak of Chikungunya in India: a cluster-randomised, double-blind, placebo-controlled trial. Indian J Res Homoeopathy 2014;8:160-165

13 Hahnemann S. Organon of Medicine. 6th ed. New Delhi: B. Jain Publishers; 2004:267

14 Kent JT. Lectures on Homeopathic Philosophy. New Delhi: B. Jain Publishers; 1977: Lecture 3

15 Schroyens F. Synthesis Treasure Edition 2009V. RadarOpus version 2.1.13

16 Zandvoort R. Complete Repertory 2019. MacRepertory, Zeus Soft Company

17 Waisse S, Oberbaum M, Frass M. The Hydra-headed coronaviruses: Implications of COVID-19 for homeopathy. Homeopathy 2020; 109:169-175

18 Kasariyans A, Sankaran R. Homeopathy for coronavirus COVID-19 infection: Dr Aditya Kasariyans and Dr Rajan Sankaran. Accessed March 29, 2020 at: https://facultyofhomeopathy.org/wp-content/uploads/2020/03/Homeopathy-for-Coronavirus-Covid-19Infection-Dr-Aditya-Kasariyans-and-Dr-Rajan-Sankaran.pdf

19 AYUSH. Advisory for Corona virus. Homoeopathy for Prevention of Corona virus Infections. Unani Medicines useful in symptomatic management of Corona Virus infection. Posted on: Jan 29, 2020 by PIB Delhi. Accessed March 12, 2020 at: https://pib.gov.in/ PressReleasePage.aspx?PRID=1600895\#

20 Rossi EG. The experience of an Italian public homeopathy clinic during the COVID-19 Epidemic, March-May 2020. Homeopathy 2020;109:167-168

21 To KLA, Fok YYY. Homeopathic clinical features of 18 patients in COVID-19 outbreaks in Hong Kong. Homeopathy 2020;109:146-162 